\title{
La biblioteca universitaria en las redes sociales: planificando una presencia de calidad
}

\author{
Por Estela Andrade y Erika Velázquez
}

Universidad de la República. Facultad de Ingeniería. Departamento de Documentación y Biblioteca. Montevideo, Uruguay

\section{Resumen}

Trata sobre la viabilidad de participación de las bibliotecas universitarias en las redes sociales. Se presentan los beneficios y oportunidades de la web 2.0 y de las comunidades en línea. Plantea un conjunto de pautas orientadas al desarrollo de un plan de trabajo que permita la implementación de sitio informativo en una red social. Se destaca las capacidades de los bibliotecólogos para optimizar esta clase de servicios.

\section{Palabras clave}

Redes sociales ; Bibliotecas universitarias ; Web 2.0

\section{Title}

The university library in social networks: planning a quality presence

\begin{abstract}
Talks about the feasibility of participation of university libraries in social networking. We present the benefits and opportunities of Web 2.0 and online communities. Poses a set of guidelines aimed at developing a work plan that enables the implementation of site information in a social network. It highlights the capabilities of librarians to optimize this type of service.
\end{abstract}

\section{Keywords}

Social networking ; University libraries ; Web 2.0

Recibido - Received: 2010-12-09

Aceptado - Accepted: 2011-03-31 


\section{Introducción}

Desde que surgieron las Nuevas Tecnologías de la Información los bibliotecólogos han hecho uso de ellas para comunicarse, compartir ideas, brindar asesoramiento y crear productos específicos.

El advenimiento de las tecnologías de la web 2.0 ha presentado nuevas oportunidades para su labor. ¿Por qué? Porque como manifestara Tim O'Reilly (quien acuñó el término):

"Las aplicaciones web 2.0 son aquellas que sacan partido a las ventajas intrínsecas de la web, ofreciendo un servicio continuamente actualizado que mejora cuanto más gente lo use, utilizando y remezclando datos de múltiples recursos... de forma tal que pueden ser utilizados por otros, creando una arquitectura de participación en red, yendo más allá de la página web 1.0 para ofrecer experiencias de usuario cada vez más ricas"

Es decir que el entorno dinámico de la web 2.0, con páginas que se actualizan constantemente y que permiten la interacción con los usuarios, da un valor agregado a la web, ya que no implica solamente el uso de tecnologías sino también la puesta en práctica de una actitud basada en los principios de compartir, reutilizar, mejora continua, consideración del usuario como fuente de información, confianza y aprovechamiento de la inteligencia colectiva. Es por todo esto que también se la denomina web social.

Tal como lo expresan Natalia Arroyo y José Merlo, el término web 2.0 designa una nueva generación de servicios web basados en una serie de tecnologías y fundamentalmente en la mayor participación y colaboración de los internautas. Estas nuevas posibilidades de información y comunicación hacen posible la construcción con menos esfuerzo y dinero- de la biblioteca 2.0, que al decir de estos autores no es más que el resultado de aplicar principios y herramientas de la web 2.0 a las bibliotecas.

"La biblioteca 2.0 es una filosofía de servicios basada en tres cosas; el deseo de cambiar y probar cosas nuevas; el deseo de reevaluar constantemente nuestras ofertas de servicios;

y finalmente, la disposición de mirar afuera de nuestro propio mundo para buscar soluciones sean estas tecnológicas o no."

(Casey, Michael, 2005, apud García Rivadulla, Sandra, 2010).

Entre los múltiples beneficios que la web 2.0 ofrece enumeramos aquellos que consideramos de valor para una biblioteca universitaria:

- Promueve la interacción al facilitar rápida y efectivamente el contacto entre los internautas aumentando la comunicación entre el staff y los usuarios, la colaboración y el conocimiento compartido.

- Permite abarcar una vasta audiencia, asistiendo a más personas en la esfera virtual que lo que sería posible en una localización física, respondiendo a sus necesidades en tiempo real.

- Brinda una nueva forma de obtener visibilidad y ofrecer servicios.

- Posee servicios gratuitos y de fácil uso inclusive por aquellos que tienen poca experiencia. 
- Actualización y publicación rápida de sus servicios superando el tiempo de demora asociado a la publicación tradicional de la web.

- Atrae a los grupos de usuarios jóvenes que tradicionalmente son menos gustosos de ir a la biblioteca física.

- Pone a disposición contenidos y brinda enlaces al sitio web de la institución y/o a otros sitios.

- Mejora la imagen de la biblioteca al brindar mayor capacidad de encontrar soluciones rápidas que satisfagan necesidades de los usuarios.

Sandra García en Actitud 2.0 nos señala al respeto:

"Lo que es indudable es que la web social se ha permeado en casi todos los ámbitos de la vida y las bibliotecas no deben estar ajenas a este cambio. Su misión es ser flexibles y adaptarse para satisfacer las necesidades de usuarios que exigen y merecen nuevos servicios"

Dadas todas las ventajas que la web 2.0 ofrece es esencial que los bibliotecólogos hagan un uso óptimo de su potencial, afrontando los desafíos de innovación constante y cambio organizacional así como la natural resistencia que suele provocar en el ámbito formal universitario.

\section{El desafío de decidir por dónde comenzar}

Actualmente uno de los servicios web 2.0 más populares lo constituyen los sitios de redes sociales. Las redes sociales en línea o sitios de redes sociales (Boyd, Ellison, 2007 apud Ortega, José Luis; Aguillo, Isidro F., 2008) pueden ser definidos como servicios basados en la web que permiten a los usuarios construir un perfil público y ofrecen las herramientas que hacen posible la interacción entre ellos, compartiendo intereses, recursos e información.

Estas plataformas se han convertido, según Sonia Fernández, en auténticas comunidades en línea donde los usuarios están en permanente contacto. Es la propia naturaleza social del ser humano la que lo hace participar de estas nuevas comunidades con el fin de satisfacer su necesidad de comunicación.

Los profesionales de la información siempre han tenido en cuenta las necesidades del usuario y precisamente por eso es que hoy las bibliotecas deberían estar presentes en las redes sociales. El uso de estas nuevas plataformas tecnológicas permite atender demandas y expectativas de los usuarios con prontitud, facilitando el desempeño del staff y la difusión de servicios.

Existen varios sitios de redes sociales, por lo que es necesario decidir cual utilizar. Para ello es conveniente:

- Determinar el sitio de red social más popular

- Considerar las preferencias de los usuarios del centro

- Delimitar el grupo de usuarios más interesado y la información a compartir

- Tener en cuenta el objetivo del sitio y las expectativas del usuario 
- Aprender de la experiencia de otros centros que ya utilicen estas herramientas

- Experimentar con los servicios de redes sociales para encontrar el que más se ajusta al servicio

\section{Uso de las redes sociales: relevamiento de datos en la Biblioteca de la Facultad de Ingeniería de la Universidad de la República}

Para poder determinar el sitio de red social más popular, en la Biblioteca de la Facultad de Ingeniería de la Universidad de la República (UdelaR), se llevó a cabo una encuesta a una muestra determinada al azar entre sus usuarios. Un $79 \%$ de los encuestados manifestó participar en las redes sociales, integrando la red social Facebook un $76 \%$.

Si bien usuarios de todas las edades manifestaron integrar redes sociales, la mayoría pertenece al rango etario de 18 a 29 años. Esto pone de manifiesto el hecho de que los chicos de hoy son nativos digitales que están inmersos en la tecnología de las comunicaciones (teléfonos celulares, reproductores MP3/MP4, internet, etc.), usan la web para "socializar", cambian la privacidad por la accesibilidad, prefieren trabajar en equipo. Para un joven que forma parte de las redes sociales on line existentes su extensión en el ámbito universitario es natural y lógica.

Como asevera Andrés Pedreño Muñoz tan lógico como que su iPod sirva para escuchar música y al mismo tiempo descargar las clases de determinadas asignaturas y escucharlas en el momento más propicio. Los motivos por los cuales los encuestados consideraron útil que la biblioteca integre una red social fueron:

- Interactuar

- Consultar en tiempo real

- Generar ámbitos de discusión entre sus integrantes

- Acceder a información

- Acercar a los estudiantes a la biblioteca y a los servicios que ella ofrece de una forma actualizada.

- Acceder a otro medio digital que permita la interacción que la página web institucional no ofrece.

Mientras tanto los encuestados que no consideraron útil la presencia de la biblioteca en las redes sociales manifestaron expresiones como:

- "Porque no es una herramienta académica, ni es confiable. Mejor sería crear un sitio web institucional"

- $\quad$ "No se me ocurre un buen motivo por el que la biblioteca tenga que estar, si lo hay, bienvenido sea"

- "Sería más necesaria una página web más interactiva que la actual (contacto directo usuario/bibliotecólogo)"

- "Lo entiendo como medio de comunicación y entretenimiento en tiempos libres"

- "El uso que le doy a las redes sociales no está relacionado con el trabajo, por ese motivo no relaciono Facebook con trabajo, aunque sé de gente que se relaciona con grupos profesionales y ahí es ventajoso. Si viera algún ejemplo de ventajas, mi opinión cambiaría"

- "No es un lugar en que buscaría información" 
Estas expresiones demuestran el desconocimiento, por parte de los usuarios, de la aplicación que se le puede dar a las redes sociales a nivel institucional. A estos usuarios se suman aquellos que no contestaron o simplemente admitieron no tener conocimiento del tema.

El uso que se le da a las redes sociales constituye otro índice de esta desinformación por parte de los usuarios ya que el $71 \%$ lo utiliza para comunicarse con amigos, el $48 \%$ para entretenimiento, un $47 \%$ para compartir imágenes y/o videos mientras que sólo un $25 \%$ lo hace para unirse a grupos profesionales y un 13\% para obtener información actualizada referente a su profesión o carrera.

Considerando los resultados de la encuesta se determinó que el sitio de red social más popular entre los usuarios es Facebook. Esto coincide con los datos de la investigación de Jorge de Oyhenard acerca de las Redes Sociales en Uruguay según la cual el $44 \%$ de los 1.300.000 usuarios de Internet tiene su perfil en Facebook. Esta popularidad, su origen en un ámbito académico, la presencia masiva de estudiantes universitarios, su apertura, facilidad de uso y herramientas que ofrece para crear aplicaciones, hacen de Facebook el servicio web preferido por los usuarios más jóvenes.

\section{Las bibliotecas académicas en las redes sociales}

Las bibliotecas universitarias que brindan servicios sensibles y receptivos deben tener en cuenta las preferencias e intereses de sus usuarios. Por ello hoy adoptan las tecnologías web 2.0 integrándose a los sitios de redes sociales. Este tipo de recurso 2.0 facilita el intercambio de información característico de nuestra profesión a la vez que, posibilita una mayor cooperación y brinda una difusión más amplia.

Los bibliotecólogos alrededor del mundo están utilizando las tecnologías web 2.0 para promocionar servicios y compartir información. Pero, ¿de qué forma lo están haciendo los profesionales de la información?

Si bien los sitios de redes sociales son fáciles de usar, los bibliotecólogos no deben hacerlo descuidadamente como el común de la gente. Ellos están acostumbrados a adaptar periódicamente sus herramientas y recursos de trabajo a los conceptos innovadores y las tecnologías emergentes sin modificar en nada sus objetivos.

El conocimiento y discernimiento en la selección y manejo de fuentes de información, así como la organización y prestación son fundamentales a la hora de aplicar las nuevas tecnologías de la información en los servicios. Por lo tanto es necesaria una actitud de apertura para adoptar herramientas potencialmente poderosas que empleándolas de modo profesional y de acuerdo a parámetros de calidad, posibilitan la creación de productos específicos.

Cuando una biblioteca universitaria pasa a formar parte de una red social, el bibliotecólogo además de ser un diseminador de información, se convierte en un comunicador que participa activamente actuando como conducto entre los internautas y la información, arbitrando la creación y comunicación de contenidos entre los usuarios. 
Según Ana Cabrera y Adrián Domínguez, los sitios de redes sociales agrupan aquellos sitios web que se proyectan no sólo como expresión de los individuos sino también de comunidades de usuarios que interactúan para intercambiar información y conocimiento. Todo sitio web es una estructura de información.

Compartiendo lo afirmado por nuestra colega Alicia García de León, este tipo de estructura de información se caracteriza por su hipertextualidad e interactividad en un escenario de acceso múltiple y masivo como es el ciberespacio. Al igual que cualquier documento debe ser pensado y concebido críticamente.

Actualmente varias bibliotecas académicas están presentes en Facebook. Esta realidad puede ser consecuencia del hecho de que otras instituciones lo están utilizando o sencillamente por "moda". Si bien es cierto que es necesario estar al día y utilizar aquellos recursos tecnológicos preferidos por los usuarios, no se debe integrar una red social presionado por la urgencia y/o la novedad sin desarrollar un plan para construir, evaluar y mantener un espacio en ese sitio.

\section{Planificando la presencia en la red social}

El estar presentes en una red social no implica simplemente poner a disposición y compartir información actualizada, es imprescindible la elaboración y ejecución de un plan de trabajo que garantice la calidad del sitio. Como menciona Alicia García de León en su investigación Etapas en la creación de un sitio web, es necesario reunir información "... tanto teórica como técnica, búsqueda de bibliografía y formativa, así como la navegación y análisis de aquellos sitios referenciales, próximos o pares, pasibles de ser emulados."

El establecimiento de objetivos claros es prioritario en toda planificación, ya que si bien pueden sufrir modificaciones a lo largo del tiempo, constituyen el punto de partida que orienta el trabajo a realizar. Al integrar una red social se comparte el objetivo general de satisfacer la necesidad de interactuar y compartir información entre los usuarios digitales. A su vez se tiene que considerar los objetivos específicos que tiene una biblioteca académica, los cuales van a determinar su perfil en la red elegida.

Una vez reunida la información y establecido los objetivos, deben ser identificados los destinatarios tanto sean estos usuarios reales como potenciales. La encuesta realizada en la Biblioteca de la Facultad de Ingeniería facilitó datos para conocer los intereses y preferencias de los usuarios, fundamental al momento de seleccionar la información a publicar. La identificación de los usuarios y sus necesidades no termina en esta etapa, sino que los contadores de visitas y estadísticas que ofrecen las redes sociales así como su característica intrínseca de interacción, permiten llevarla a cabo en forma continua.

\section{Como estructurar y presentar los contenidos en la red social}

Identificados los usuarios se establecen los contenidos que se presentarán en el sitio a fin de satisfacer sus demandas y cumplir con los objetivos fijados. A la hora de estar presentes en una red social es fundamental identificar la biblioteca a través de su nombre completo, el URL de la página web institucional, la dirección de correo electrónico y los responsables. 
Según Alicia García de León la información debe ser presentada de acuerdo a los requerimientos propios de la escritura web:

"Los textos...deben ser breves, concisos y puntuales, muy estructurados que permitan una visión general y la opción a ampliaciones o profundizaciones mediante enlaces. Deben tener títulos y subtitulos claros y directos, que permitan gran movilidad, que vinculen a otros textos dentro y fuera del sitio web, o sea que... permitan un abordaje dinámico y directo"

Al presentar los contenidos en una red social se deben tener en cuenta siempre los siguientes criterios:

- Selección: Los contenidos publicados, además de satisfacer las demandas de los usuarios y contemplar los objetivos del sitio, deben provenir de fuentes confiables. El bibliotecólogo, como profesional de la información, es quien deberá realizar esa cuidadosa elección y supervisar el origen de los contenidos que publiquen los usuarios, realizando las pertinentes aclaraciones en los casos que sea necesario.

- Ortografía: Se debe controlar que los textos que se publiquen no tengan faltas ortográficas ni errores gramaticales.

- Citación: Todo documento publicado ya sea texto, foto, cuadro o gráfico debe ser citado correctamente.

- Estructura de la información: No es suficiente poner a disposición información de interés y actualizada, debe ser presentada de acuerdo a un criterio lógico. La forma como se estructura la información es tan importante como la información en sí misma, la cual debe ser organizada mediante una estructura jerárquica en la que se establecen áreas y relaciones entre las mismas. A esa estructura conceptual nuestra colega Alicia García de León la denomina ordinograma.

- Navegabilidad: El crear pestañas apropiadas al ordinograma establecido por la biblioteca hace posible una buena navegación del sitio. La estructuración de los contenidos debe ser clara de modo de facilitar los procedimientos de búsqueda. Así el usuario podrá acceder a los contenidos de su interés siguiendo las indicaciones brindadas en el propio sitio y a través del menor número de clics posible, no mediante ensayo y error, que además de ocasionar pérdida de tiempo puede llegar a ser tedioso.

No hay que olvidar tampoco que, la estructuración además de clara debe ser simple, pues si se ofrecen buenos contenidos distribuidos en una estructura compleja se corre el riesgo que el usuario no la visite y prefiera acceder a otros sitios de menor calidad pero más accesibles.

Muchas de las bibliotecas que están presentes en Facebook utilizan solamente las pestañas predeterminadas, lo que demuestra la inexistencia de una estructuración previa de la información así como un manejo limitado de las posibilidades que brinda dicha red social. 
Se debe controlar también que los contenidos no se reiteren en distintas partes del mismo sitio, como suele suceder en las páginas de Facebook de varias bibliotecas académicas. Esta situación puede prestar a confusión, generar aburrimiento, o dificultar el hallazgo de lo que interesa o es útil al internauta.

- Diseño: Todo sitio web posee un diseño que constituye su expresión gráfica permitiendo su visualización y navegación. Se realiza de acuerdo a los objetivos y contenidos del sitio, cuidando la estética y asegurando el correcto suministro, uso y transferencia de la información.

- Enlaces: Si una biblioteca pretende una presencia de calidad en una red social debe realizar una selección on criterio de los enlaces, teniendo en cuenta la estabilidad de la fuente que lo suministra y el grado de actualización del sitio. Los enlaces seleccionados al ser presentados deben ser citados correctamente y actualizados en forma continua. La selección y presentación de enlaces y no la cantidad es lo que importa a la hora de evaluar un sitio.

- Normativa: Si bien la privacidad es tenida en cuenta por los servicios de redes sociales que ofrece la web mediante el uso de múltiples opciones configurables, es conveniente que el administrador de la cuenta de Facebook de la biblioteca académica en cuestión elabore y publique una normativa de participación. Se comunicará, por ejemplo, que el incumplimiento de alguna de las normas establecidas supondrá la eliminación del comentario del participante.

- Promoción: Todas las actividades y servicios que la biblioteca considere conveniente se promocionarán a través de la red social, así como mediante el uso de etiquetas de los recursos que pueden ser de interés de los fans.

En el caso específico de la Facultad de Ingeniería uno de los motivos por lo que se decidió integrar una red social es el publicitar de una manera rápida y efectiva la puesta en funcionamiento de un software integrado de gestión de bibliotecas (Aleph) en la UdelaR. A su vez se llevará a cabo la promoción de la red social que integre la biblioteca mediante la creación de un link desde el sitio web de la institución.

- Mantenimiento y actualización: Es imprescindible que los encargados de la biblioteca en el sitio de red social realicen una actualización periódica de los contenidos y links. Por ejemplo, si alguien plantea una interrogante en el muro hay que responderle, si han colgado links hay que supervisar su vigencia.

El éxito o fracaso en el uso de las aplicaciones web 2.0 depende, como afirma Marta Nogueira, de la capacidad que tienen las organizaciones de mantener un uso activo de las mismas. 


\section{Evaluar la presencia de la biblioteca académica en la red social}

La evaluación es esencial en la construcción, ejecución y mantenimiento de un sitio de red social. Una vez creado la página en el sitio hay que responder a interrogantes como:

- ¿Cumple con los objetivos establecidos?

- ¿Presenta los contenidos pensados? ¿se han estructurado con claridad y calidad?

- ¿Tiene un diseño gráfico apropiado?

- ¿Funcionan todos los enlaces publicados?

- ¿Tiene dificultades de operatividad?

Puesto en marcha el sitio debe evaluarse su impacto. La propia red social es la que brinda información acerca de la cantidad de veces que es visitada, por quiénes y cuando.

La interactividad importa no sólo por lo que significa en sí misma sino también porque permite detectar aciertos, dificultades y expectativas. La evaluación se efectúa constantemente verificando lo propuesto en las etapas previas, permitiendo así la revisión, adaptación y mejora continua.

Teniendo en cuenta el ciclo PDCA, también conocido como "Círculo de Deming o circulo de Gabo", modelo conceptual de actividades interdependientes que influyen en la calidad de un producto; la planificación se lleva a cabo en las distintas fases que van desde identificación de las expectativas de los usuarios y establecimiento de objetivos hasta su evaluación. Este ciclo se recorre constantemente, es decir que luego de poner en marcha el sitio en la red social se debe considerar como evoluciona y así obtener insumos para la mejora de su próxima versión. De no cumplirse se corre el riesgo de tener una presencia de mala calidad en la red social.

\section{Conclusiones}

- Los bibliotecólogos han estado utilizando para comunicarse, compartir ideas y brindar asesoramiento las nuevas tecnologías de la información. El advenimiento de la web 2.0 ha presentado nuevas oportunidades al promover la interactividad entre los usuarios mediante servicios gratuitos que solo requieren inversión de tiempo del staff y que permiten atender demandas y expectativas con prontitud.

- Es fundamental superar la natural resistencia existente en los ámbitos académicos a estar presentes en la web 2.0, haciendo de la red social un instrumento más de comunicación, divulgación y evaluación de sus servicios.

- Al momento de decidir en que red social estar presente se tiene que determinar el sitio más popular entre sus usuarios, considerar sus preferencias y expectativas, contactar con profesionales de otras bibliotecas que hayan adoptado servicios similares, conocer los objetivos de las redes sociales existentes en plaza y experimentar con ellas.

- $\mathrm{Al}$ adoptar estas nuevas herramientas hay que tener presente que no funcionan por si solas, es necesaria la presencia de un profesional de la información que tenga 
a cargo la estructuración, presentación, control y actualización de los contenidos publicados.

- La calidad del sitio de una biblioteca académica en una red social es garantizada mediante un plan de trabajo que abarque la determinación de objetivos, identificación de usuarios y sus necesidades, construcción, diseño, mantenimiento, evaluación y la consiguiente retroalimentación que permitirá su puesta al día.

- El valor de los sitios de redes sociales radica en el uso que se le otorgue. Tienen, como cualquier otra herramienta, fortalezas y debilidades que el bibliotecólogo debe tener en cuenta siempre que pretenda elaborar productos de calidad.

\section{Bibliografía}

A guide to using web 2.0 in libraries. (2009). En: The Scottish Library and Information Council. Disponible en:

< http://www.slainte.org.uk/files/pdf/web2/web2Guidelinesfinal.pdf $>$

Arroyo, Natalia. (2009). Panorama general de las redes sociales. Disponible en: $<$ http:/ / comunidad20.sedic.es $/$ ? $\mathrm{p}=231>$

Arroyo Vázquez, Natalia. (2007). ¿Web 2.0? ¿web social? ¿qué es eso?. En: Educación y Biblioteca, 161: 68-74. Disponible en:

<http://eprints.rclis.org/11752/1/EYB_NA07.pdf>

Arroyo Vázquez, Natalia; Merlo Vega, José A. (2007). La biblioteca como usuaria de la web 2.0. En: 10as. Jornadas Españolas de Documentación, FESABID. Santiago de Compostela, España. Disponible en:

$<\underline{\text { http://www.cedus.cl/files/Arroyo\&Merlo_FESABID07.pdf }>}$

Baeza Yates, Ricardo; Rivera Loaiza, Cuauhtémoc; Velasco Martín, Javier. (2004). Arquitectura de la información y usabilidad en la web. En: El profesional de la información, 13 (3): 168-178. Disponible en:

$<$ http://elprofesionaldelainformacion.metapress.com/app/home/contribution.asp?re ferrer=parent\&backto=issue,1,13;journal,37,71; homemainpublications, 1,1;>

Cabrera Facundo, Ana Margarita; Coutín Domínguez, Adrián. (2005). Las bibliotecas digitales. Parte I: Consideraciones teóricas. En: Acimed, 13 (2). Disponible en: $<\underline{\text { http://bvs.sld.cu/revistas/aci/vol13_2_05/aci04205.htm }>~}$

Celaya, Javier. (2009). Definir la presencia de las bibliotecas en las redes sociales. Disponible en: <http:/ / comunidad20.sedic.es / ?p=240>

Fernández, Sonia. (2008). Redes sociales: ¿Fenómeno pasajero o reflejo del nuevo internauta? En: Telos: Cuadernos de Comunicación e Innovación, 76. Disponible en: $\leq$ http://sociedadinformacion.fundacion.telefonica.com/telos/articulocuaderno.asp@i $\underline{\text { darticulo }=11 \& \text { rev }=76 . \mathrm{htm}>}$ 
García De León, Alicia. (2002). Etapas en la creación de un sitio web. En: Biblios Revista Electrónica de Bibliotecología, Archivología y Museología, 4 (14): 1-17. Disponible en: $<\underline{\text { www.redalyc.org }>}$

García De León, Alicia; Garrido Díaz, Adriana. (2002). Los sitios web como estructuras de información: un primer abordaje en los criterios de calidad. En: Biblios Revista Electrónica de Bibliotecología, Archivología y Museología, 3 (12) 1-16. Disponible en: $<\underline{w w w}$.redalyc.org $>$

García Rivadulla, Sandra. (2010). Actitud 2.0: Usos de la web social en las bibliotecas universitarias uruguayas. Disponible en:

$<$ http:/ / alfinuruguay.blogspot.com/2010/06/actitud-20-usos-de-la-web-social-en$\underline{\text { las.html }>}$

Leiva Aguilera, Javier. (2009). Qué dicen por ahí... Disponible en:

<http://comunidad20.sedic.es/?p=243>

Margaix Arnal, Dídac. (2008). Las bibliotecas universitarias y Facebook: cómo y por qué estar presentes. En: El profesional de la información, 17 (6): 589-601. Disponible en: $<$ http://elprofesionaldelainformacion.metapress.com/app/home/contribution.asp?re ferrer=parent\&backto=issue,2,16;journal,10,71; homemainpublications,1,1;>

Margaix Arnal, Dídac. (2007). Conceptos de web 2.0 y biblioteca 2.0: origen, definiciones y retos para las bibliotecas actuales. En: El profesional de la información. 16 (2): 95-106. Disponible en:

$<$ http://elprofesionaldelainformacion.metapress.com/app/home/contribution.asp?re ferrer=parent\&backto=issue,2,15;journal,20,71; homemainpublications, 1,1;>

Mirescu, Kathy. (2009). Arquitectura de la Información: Un Modelo para la Bibliotecología del Siglo XXI. Disponible en:

<http://spanish.argentina.usembassy.gov/evento_mirescu/mir.pdf $>$

Nogueira, Marta. (2010). Archives in Web 2.0: New Opportunities. En: Ariadne, 63.

Disponible en: <http://www.ariadne.ac.uk/issue63/nogueira/>

Ortega, José Luis; Aguillo, Isidro F. (2008). Análisis estructural de una red social en línea: la red española de Flickr. En: El profesional de la información, 17 (6): 603-610. Disponible en:

<http:// www.elprofesionaldelainformacion.com/contenidos/2008/noviembre/03.ht $\underline{\mathrm{ml}}>$

Pedreño Muñoz, Andrés. (2007). Tecnologías de educación universitaria. Disponible en: <http://studium.universiablogs.net/apm>

Sánchez Carballido, Juan Ramón. (2008). Perspectivas de la información en Internet: ciberdemocracia, redes sociales y web semántica. En: Zer, 13 (25): 61-81. Disponible en<http://www.ehu.es/zer/zer25/zer25-3-sanchez-carballido.pdf> 
Torre De La, Aníbal. (2009). Definición de Web 2.0. Disponible en:

$<$ http://www.adelat.org/index.php?title=conceptos_clave_en_la_web_2_0_y_iii\&mor $\mathrm{e}=1 \& \mathrm{c}=1 \& \mathrm{tb}=1 \& \mathrm{pb}=1>$

Turnbull Muñoz, M. En C. Federico. (2010). Retos y oportunidades para el bibliotecólogo ante las nuevas tecnologías de información. Disponible en: <http://148.226.9.79:8080/dspace/bitstream/123456789/7697/1/mem29pag260265.pdf $>$

Uribe Tirado, Alejandro; Echavarría Ramírez, Andrés Felipe. (2008). Facebook como red de profesionales de bibliotecología, documentación y archivística en Iberoamérica. En: El profesional de la información, 17 (6): 670-676. Disponible en:< http://elprofesionaldelainformacion.metapress.com/app/home/contribution.asp?refe $\underline{\text { rrer=parent\&backto=issue,11,16;journal,10,71; homemainpublications,1,1;> }}$

Van Der Henst S, Christian. ¿Qué es la web 2.0? Disponible en: <http://www.maestros delweb.com/editorial/web2/>

Wikipedia. (2011). Círculo Deming. Disponible en:

<http://es.wikipedia.org/wiki/C\%C3\%ADrculo_de_Deming>

\section{Datos de las autoras}

\section{Estela Carmen Andrade Andrade}

Biblioteca Referencista. Universidad de la República. Facultad de Ingeniería.

Departamento de Documentación y Biblioteca. Montevideo, Uruguay eandrade@fing.edu.uy

\section{Erika Jimena Velazquez Guerrero}

Biblioteca Referencista. Universidad de la República. Facultad de Ingeniería.

Departamento de Documentación y Biblioteca. Montevideo, Uruguay

erivelaz@fing.edu.uy 